\title{
The Study of Cable Operation Fault Monitoring Based on All-fiber Technology

\author{
Xiaodong Liu ${ }^{1}$, Defeng Chen ${ }^{2}$, Nan Sun ${ }^{3^{*}}$ \\ ${ }^{1}$ State Grid Nanjing Power Supply Company, Nanjing, Jiangsu, China, 210000 \\ ${ }^{2}$ Nanjing Suyi Shiye Company, Nanjing, Jiangsu, China, 210000 \\ ${ }^{3}$ Bandweaver Technologies Company, Beijing, China, 100000 \\ hunter2011@foxmail.com
}

Keywords: Cable; Operation Fault Monitoring; All-fiber Technology

\begin{abstract}
The on-line detection of temperature and strain is the necessary measure for ensuring the safety and stability of the system during power cable's operation .The distributed fiber optic sensing detecting system for on-line monitoring high-voltage cable is presented. The measurement equipments used in the detecting system are described in detail. The new type of sensing optical fiber, carbon coated fiber, which is suitable to be used in the complicated environment of electricity system for the on-line measurement of temperature and strain is investigated .And the installation method for this type of sensing optical fiber is discussed. The distributed fiber optic sensing detecting system will have bright application prospect in the on-line detection of high-voltage transmission system.
\end{abstract}

\section{Introduction}

Power system online health monitoring high-voltage cables can be effectively avoided cable faults, to ensure security of supply and stable operation of the power system. Rally or soil temperature changes and external environment caused by subsidence, can make high voltage cables produce vertical or horizontal deformation, severe cases may lead to high-voltage cables armored, lead (aluminum) sheath rupture, or even broken; the same time, high-voltage cables the fires often happen, it is necessary to install high-voltage cable systems for real-time accurate strain and temperature on-line testing to ensure the safe operation of high voltage cables.

Whether it is the traditional method of temperature measurement point temperature sensing element (such as thermocouples) mounted on the cable focus detection site, or the use of quasi-distributed fiber grating temperature measurement methods, are only on the temperature of the cable local position is detected, and Unable to carry out the whole line-line monitoring. In recent years, with the development of optical fiber application technology, distributed fiber optic sensing system only with the high voltage cable laying an optical fiber can provide information on the operating temperature and strain the entire cable, with no interference, no radiation, simple construction, safety sex characteristics, can provide a scientific basis for temperature and strain detection and safe operation of the cable, so as to effectively prevent the occurrence of high-voltage cables accidents. Currently distributed fiber optic sensing systems have been widely used in the cable industry.

\section{The Constitution of Fiber Optic Sensing System}

Distributed optical fiber sensing technology is based on the principle of Raman scattering is mainly used for detecting the temperature of the line, high voltage cable temperature measurement information is relatively simple, by working wave length (about $0.9 \mu \mathrm{m}$ ), the scattered light signal is weak constraints, sensing distance is shorter. And based on the unique principle of distribution of Brillouin scattering fiber-optic sensing technology that enables simultaneous measurement of temperature and strain, and the Brillouin scattered light signal is stronger; single mode optical fiber as a sensing fiber, the operating wavelength selectable at $1.3 \mu \mathrm{m}$ or $1.55 \mu \mathrm{m}$, the wavelength of the 
optical signal attenuation and dispersion is small, the sensing distance can be up to tens of kilometers, thus achieving long-distance high-voltage cables testing. Since the high-voltage cable fault showed both temperature and strain changes exhibits, so the use of Brillouin distributed fiber optic sensing technology, can realize online detection and fault points on the entire cable line fast positioning, to achieve the temperature and strain at the same time detection and measurement of simple, accurate positioning.

Real-time information to detect high-voltage cable detection system is by collecting data demodulation unit acquired temperature, strain information, and to temperature, strain distribution curve form displays measurement data acquisition. System design temperature resolution of $0.1{ }^{\circ} \mathrm{C}$, the spatial resolution of $1 \mathrm{~m}$, measured distance of $30 \mathrm{~km}$, can be set to a multi-stage fixed temperature alarm, and for different field environments amendment to increase the flexibility of the system. Since the sensing fiber is immune to electromagnetic interference, instant strength current, humidity and corrosion, and ensure the stability of the system.

\section{Testing Equipment}

Constituting high voltage cable detection system is comprised by: a light source, a light pulse forming unit, a photo detector unit and data processing unit. The center wavelength of the light source is a high voltage cable detection system, an important parameter. Different wavelengths of light in the sensing fiber dispersion and attenuation of different Brillouin scattering intensity and frequency shift are different. To improve the measurement accuracy of Brillouin scattering efficiency and systems should narrow line width, high-power (generated by the case does not stimulated Brillouin scattering) of tunable semiconductor lasers as light sources. We choose the center wavelength of the light source $1.55 \mu \mathrm{m}$, a line width of $1.6 \mathrm{MHz}$, the output power of $15 \mathrm{~mW}$.

Light pulse forming unit by the acousto-optic modulator, amplifier, and filter. The unit can achieve continuous optical modulation pulse light, ASE noise amplification and filter out other functions of the optical signal.

Photo detector unit includes a photoelectric detector, microwave local oscillator, mixer, bandpass filter. Brillouin scattering light amplified and filtered into the photo detector with the reference light heterodyne detection, the output difference frequency signals (Brillouin frequency shift signal), due to the high frequency of the signal cannot get an accurate signal processing, and therefore the required frequency to a lower frequency, and then enters the data processing unit performs analysis processing signal frequency.

The data processing unit in data acquisition card output signal storage and processing, to complete a measurement information curve. Due to loss and noise detection system for the entire test, the need for multiple measurements, the data accumulated average and wavelet de-noising processing to obtain better reflection of the true temperature of the high-voltage cable lines and strain curves. The information processing based on virtual instrument Labview8 .6 basic platform.

\section{Sensing Fiber}

Distributed fiber optic sensing system of high voltage, high current power system operation and other complex environment, the sensing fiber path is simultaneously used as a sensor and signal transmission channels, so far away from the measurement site of the terminal to obtain the corresponding information to enable terminal completely avoid the interference of strong electromagnetic fields. For the $220 \mathrm{kV}$ high-voltage cables, cable diameter sensor will affect its mechanical properties, but also restricts their thermal conductivity, in order to make the cable has a good thermal performance in good mechanical properties, you must select a new sensing fiber.

Carbon hermetically coated optical fiber selection is critical to high voltage cable detection system. High-strength carbon hermetically coated optical fiber attenuation will affect the measurement accuracy and range of distributed fiber optic sensing system, so it should have excellent optical properties. According to field application experience, the attenuation coefficient of carbon hermetically coated optical fiber should be controlled in $0.6 \mathrm{~dB} / \mathrm{km}$ or less, anti-hydrogen 
carbon hermetically coated optical fiber (moisture) permeability factor should reach 99.9 percent, fatigue factor $\mathrm{N}$ values between 350 to 1000, fiber optic life of up to 20 years.

Tube current, some foreign manufacturers processing method used is the first one with good flexibility hollow stainless steel tube or tubes installed inside the cable or fixed surface after installation of the cable in, and then blown into the hollow fiber in so that the sensor is not affected by the installation of fiber optic cable manufacturing and installation process, and can be very easy for the fiber to be replaced. This method ensures that the sensing fiber bending effects without any due process or installation cable caused. Usually in the high-voltage cable detection system, the need to close a plurality of fiber optic sensor buried high voltage cable winding for each position on the cable periphery comprehensive monitoring, information reflecting the real high-voltage cable line. Taking into account factors such as the system cost, high-voltage cable detection systems generally use four carbon hermetically coated optical fiber laying sensor installation. Figure 1 shows the arrangement and location of these four sensing fiber, they are labeled $L_{1}, L_{2}, L_{3}$ and $L_{4}$, their position on the cable section are $\theta_{1}, \theta_{2}, \theta_{3}$ and $\theta_{4} . L_{1}, L_{2}, L_{3}$ and $L_{4}$ are uniformly distributed in the surface of high-voltage cable and each optical fiber is relatively parallel to a direction along the high-voltage cables helically stranded. Laying carbon coated optical fiber sensing fiber should pay attention to bend radius cannot be too small, otherwise it will cause bending loss optical fiber. Especially when the adit shaft construction, in order to minimize bending loss, bending radius of the fiber should be greater than 100 times the fiber diameter and that is bending radius of not less than $30 \mathrm{~cm}$.

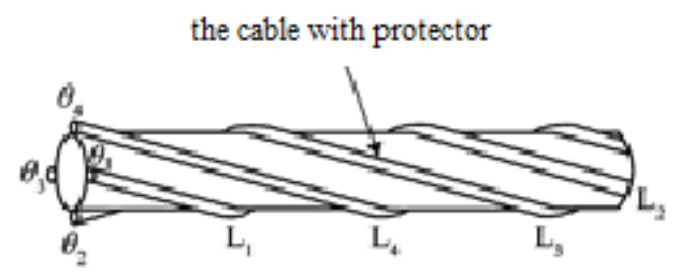

Fig. 1 The sensing fiber installation diagram

Key high-voltage cable detection system is how to improve the measurement accuracy of the temperature and strain. Factors affecting measurement accuracy of high voltage cable detection system are: the incident light intensity, the sensing fiber installation, system noise, Brillouin scattering coefficient, the number of superimposed multiple measurements, temperature and strain resolution, etc., and therefore the sensing fiber installation way affect the accuracy of the actual measurement still further research on the high-voltage cable detection system.

\section{Conclusion}

Electrically insulating fiber itself and the inherent broadband can have long-distance transmission, etc., so that the distributed fiber optic sensing system terminal from the measurement site, avoiding the harsh environment. Carbon hermetically coated optical fiber corrosion resistance, high temperature resistance, fatigue performance, can transfer the cable under test temperature and strain information, easy to install and use special occasions, high temperature and pressure to avoid damage to the optical fiber cable failure. Therefore, this fiber optic sensing principle is based on Brillouin scattering to study the use of carbon hermetically coated optical fiber as a sensing optical fiber line testing system, improve power system reliability have considerable prospect.

\section{References}

[1] Juan Z. Other Applications Distributed Optical Fiber Temperature Measurement Technology in Cable Temperature Detection. Shandong Science, 2008, 21 (6): 50-54.

[2] Zhang-Ji S. Brillouin Sensing System Used in High-voltage Cable Detection. Power System Communication, 2008, 29 (12): 55-58. 
[3] Zhang S. Application Brillouin Sensing Technology to Monitor Cable Insulation Aging. Optical Communication Technology, 2008, 32 (10): 49-52.

[4] Cheng-Xiao W. Brillouin Distributed Temperature and Strain Measurement System Simultaneously Research[D]. Baoding: North China Electric Power University, 2008. 